\title{
Evaluación docente universitaria: Hacia una perspectiva unificada
}

\section{[Evaluating university teaching: Towards a unified perspective]}

\author{
Rodrigo A. Asún \& Claudia Zúñiga \\ Universidad de Chile
}

\begin{abstract}
Resumen
La calidad de la docencia universitaria se evalúa principalmente a través de cuestionarios aplicados a los estudiantes, los que no siempre surgen de un enfoque teórico sólido. Con el fin de avanzar en la construcción de un modelo teórico que dé sustento a al desarrollo de instrumentos de evaluación, tres fuentes de demandas hacia la docencia universitaria: demandas de los estudiantes, demandas del modelo de formación por competencias y demandas de la tradición de investigación cuantitativa en docencia. A partir del análisis de estas fuentes, se construyó una conceptualización integrada de la calidad de la docencia universitaria que se espera contribuya como herramienta para el desarrollo de mejores instrumentos de evaluación de la calidad de la docencia universitaria.
\end{abstract}

Palabras clave: Competencias docentes, Evaluación de profesores, Calidad docente, Educación superior, Encuestas, Estudiantes universitarios.

\begin{abstract}
Currently, university teaching quality assessment generally uses student's responses to multiple choice questionnaires, which must be developed based on a strong theoretical approach. However, this approach is uncommon in questionnaire development. Thus, contribute to develop a theoretical model to support questionnaire development, current study describes and compares three sources of demands towards university teaching: students demands, the competency-based teaching model demands, and empirical research tradition in the field of quantitative assessment. By the mean of the analysis of these sources, we operationalized the concept of "competent teacher" which may serve as a tool for the development of better assessment tools of university teaching quality.
\end{abstract}

Keywords: Teaching competences, Teacher evaluation, Quality of teaching, Higher education, Survey, High school student.

Financiamiento: La presente investigación fue realizada mediante el financiamiento del proyecto SOC08/06-2 dela Vicerrectoría de Investigación y Desarrollo y la Facultad de Ciencias Sociales de la Universidad de Chile.

Contacto: La comunicación sobre este artículo debe ser enviada a Rodrigo A. Asún, email: rasun@uchile.cl. 


\section{INTRODUCCIÓN}

La investigación empírica sobre calidad de la docencia universitaria tiene una larga historia y amplia productividad. Uno de los hitos que suelen señalarse es que ya en 1927, Remmers diseñó y aplicó en la Universidad de Purdue, EEUU, el primer cuestionario para la evaluación de profesores de educación superior (García-Garduño, 2000). Los inicios sistemáticos de este campo de estudio se pueden situar en la década de los 60 (Onwvegbuzie et. al., 2007), alcanzando su consolidación a finales de los 70 , con la aparición de numerosos estudios y los primeros meta-análisis (e.g., Cohen, 1981). En la actualidad, estamos en presencia de un área de estudio totalmente afianzada, pues se publican decenas de investigaciones cada año y un meta-análisis cada 5-10 años.

En la literatura especializada pareciera haber consenso respecto a que la evaluación de la calidad de la docencia universitaria es una tarea compleja, que requiere, en primer lugar, una clara definición de lo que constituye un "buen docente" o un "docente efectivo" y, en segundo lugar, utilizar múltiples fuentes de información que se complementen y solapen para aumentar la validez de las estimaciones (Salazar, 2010). Sin embargo, se puede constatar que la mayoría de las investigaciones publicadas sobre la materia se limitan a presentar y discutir resultados de cuestionarios de evaluación de la docencia universitaria aplicados a estudiantes.

De igual forma, se puede observar que, en la mayor parte de las instituciones de educación superior, la evaluación de la calidad de la docencia se realiza mediante la aplicación de este tipo de instrumentos, acompañados en escasas ocasiones por alguna otra técnica (Salazar, 2008; Simpson \& Siguaw, 2000).

Lo anterior no es producto de un consenso respecto a que las respuestas de los estudiantes a instrumentos cuantitativos sean la fuente de información más válida sobre el desempeño de sus profesores. Por el contrario, un argumento habitual en contra de esta estrategia es el carácter incompleto de la mirada de los estudiantes, y para resolver este problema se han sugerido modelos evaluativos más complejos (Stake, Contreras, \& Arbesú, 2011; Tejedor, 2003) basados en redes de indicadores verificables externamente, que involucren más actores (e.g., pares, jefes, autoevaluación) y que no sólo midan la satisfacción del estudiante, sino que evalúen también el proceso de la clase por ejemplo, a través de observadores expertos, o el producto de la docencia a través de evaluaciones del aprendizaje efectivo de los estudiantes.

La debilidad de este planteamiento integrado son los altos costos que requiere su implementación. Por ello, lo que prima hoy en la mayoría de las universidades, es que la efectividad docente se mide, principal o exclusivamente, a través de encuestas a los estudiantes. Las escasas excepciones en general están aún en desarrollo (e.g., Contreras, 2010), y los principales complementos son la inclusión 
de encuestas de autoevaluación o la evaluación por jefes directos.

\section{Validez de las encuestas de evaluación docente}

Si bien se ha establecido con cierta certeza que los resultados de estas encuestas son válidos, pues explican entre el 30 y el $40 \%$ de los aprendizajes de los estudiantes (Marsh \& Roche, 1997), estas conclusiones no convencen a todos, cuestionándose tanto la presencia de sesgos en los resultados (García-Garduño, 2000), dentro de los cuáles el que más polémica ha generado es la indulgencia de los profesores en las evaluaciones (Greenwald \& Gillmore, 1997); como diferencias en la comprensión e interpretación de los ítems por parte de los estudiantes, lo que hace muy complejo interpretar sus respuestas (Kalayci, 2009).

Aunque el debate aún está abierto, actualmente la evidencia más sólida indica que la mayor parte de los sesgos estudiados no afectan o tienen impactos poco apreciables en los resultados, siempre que se utilicen instrumentos de buena calidad.

En este sentido, existen estudios que indican que cuando el diseño de los instrumentos se guía por un modelo teórico sólido y se siguen reglas metodológicas básicas, se obtienen instrumentos razonablemente válidos $\mathrm{y}$ confiables (Aleamoni, 1999; D'Apollonia \& Abrami, 1997).

Pese a lo anterior, es frecuente que el diseño de instrumentos para medir la calidad de la docencia universitaria, sea realizado en casi completa ausencia de un marco teórico sobre el proceso de enseñanza-aprendizaje que permita, a partir de una representación de como éste ocurre, ayudar a establecer sus objetivos y metas, delimitando así las conductas esperadas en el docente (Contreras et. al., 2007; Spooren, Martelmans, \& Denekens, 2007). En un estudio que analiza varias investigaciones sobre calidad de la enseñanza en educación superior, Guzmán (2011) concluye que la mayoría de las investigaciones revisadas carecen de fundamento teórico y evidencian un empirismo ingenuo al dar por sentado que el tema es unívoco y no requiere ser abordado desde alguna perspectiva que permita confrontarlo y validarlo.

En el presente trabajo, a partir de la revisión de más de 60 artículos en los que se describe el diseño o la aplicación de cuestionarios de valoración de la docencia, hemos podido constatar que en general, no hay consenso en la forma de definir operacionalmente lo que se entiende por enseñanza de calidad, ni tampoco se despliegan suficientes esfuerzos por caracterizarla teóricamente.

En relación a los conceptos utilizados por los diseñadores de las encuestas utilizan frecuentemente los conceptos de "buen docente", "docente competente", "docente excelente" y "docente efectivo" para describir la buena enseñanza; y en general, pueden distinguirse dos nociones que recorren y agrupan estas conceptualizaciones. En primer lugar, un grupo de investigadores se concentra en el docente y su conducta, y se refiere con esas etiquetas al profesor universitario que posee una serie de habilidades que abarcan tanto el trabajo dentro 
Asun, R. A., \& Zuñiga, C. (2017). Evaluación docente universitaria: Hacia una perspectiva unificada. Revista de Sociología 32(1), 50-70. doi: 10.5354/0719-529x.2017.47885

del aula (e.g., capacidades de interacción con los alumnos, dominio de la didáctica y comunicación), la capacidad de planificar escenarios educativos, dar adecuada atención a sus alumnos, dominar técnicas de seguimiento y evaluación de los aprendizajes, entre otras. Posiblemente, la principal debilidad de este enfoque ha sido que ese conjunto de conductas generalmente no son delimitadas desde un marco teórico explícito, sino más bien desde la intuición de un equipo de expertos desarrolladores del test, que en algunos casos se apoyan en investigaciones cualitativas con estudiantes y profesores (cf. Greimel-Fuhrmann \& Geyer, 2003).

Otro grupo de autores, que usualmente prefiere hablar de "docentes efectivos" (cf. Engelland, 2004), se concentra más en la conducta de los estudiantes que en la de los docentes, planteando que, en definitiva, sean cuales sean las acciones de los profesores, serán mejores aquellos que logren que los educandos aprendan más. Desde esta perspectiva no hay a priori un esfuerzo explícito por definir las conductas que conducirían a esa efectividad pedagógica, y más allá de señalar la existencia de dos "paradigmas" sobre el rol del docente (Ruiz, 2005) -el "clásico" como profesor transmisor de conocimientos y el "moderno" como facilitador-, la definición de la docencia efectiva se ha realizado por la vía operacional, es decir, delimitando las áreas o propiedades que debe tener un buen profesor al momento de redactar o justificar los ítems del test. Lo habitual ha sido intentar validar estos instrumentos posteriormente, utilizando procedimientos estadísticos -usualmente basados en la consistencia interna de los ítems- y su capacidad para predecir mayores aprendizajes en los estudiantes.

Una dificultad que tiene este acercamiento inductivo es que no ha tendido a producir ningún acuerdo sobre cuáles o cuántas serían las dimensiones o áreas que componen la buena docencia. Se han presentado propuestas de nueve dimensiones (cf, Marsh, 1991), tres dimensiones (cf. Patrick y Smart, 1998), existiendo incluso una propuesta de unidimensionalidad (Abrami \& d'Apollonia, 1991).

En suma, se observa una importante debilidad en el diseño de instrumentos cuantitativos dirigidos a estudiantes universitarios para evaluar la calidad de la docencia: la mayoría han sido construidos sin marcos teóricos sólidos y/o no han delimitado adecuada y explícitamente qué se quiere decir con "docencia de calidad". En estas condiciones, no es sorprendente que desde muchos sectores se dude de la validez de los resultados de dichos instrumentos.

El presente artículo pretende aportar a resolver esta dificultad construyendo una propuesta de conceptualización de la calidad de la docencia universitaria sólidamente fundada, que facilite el diseño de encuestas válidas y confiables.

Para el logro de este objetivo se utilizará el concepto de "docente competente" (Elton, 1996), asumiendo que esta denominación delimita suficientemente las conductas docentes como para saber cuándo estamos en presencia de un profesor que desempeña adecuadamente su rol, 
Asun, R. A., \& Zuñiga, C. (2017). Evaluación docente universitaria: Hacia una perspectiva unificada. Revista de Sociología 32(1), 50-70. doi: 10.5354/0719-529x.2017.47885

incluyendo la noción de "competencias", que es muy relevante en nuestra propuesta de conceptualización.

Entonces, la pregunta inicial que requiere plantearse en este estudio es: ¿desde qué perspectivas y con qué metodología se puede definir el concepto de docente universitario competente?

\section{Perspectivas a considerar}

Cada teoría sobre el proceso de enseñanzaaprendizaje plantea acentos y delimitaciones diferentes sobre lo que es una docencia de calidad. Además, cada actor involucrado en este proceso posee su propia mirada "intuitiva" al respecto (Gómez \& Alzate, 2010; Solar \& Díaz, 2009).

En esta pluralidad, hemos seleccionado como soporte teórico para nuestra conceptualización el enfoque de formación por competencias, opción que se fundamenta en dos argumentos: (i) este modelo goza de actualidad en el debate educativo universitario contemporáneo, siendo el soporte de los mayores esfuerzos transformadores de los últimos tiempos (e.g., el proceso de Bolonia); y (ii) más allá de las críticas conceptuales que sostienen la poca novedad, ambigüedad o inconsistencia interna de este enfoque (Barriga, 2005; Gómez \& Alzate, 2010), el enfoque de formación por competencias amplía el foco de las demandas al docente universitario, exigiéndole centrarse más en el estudiante, sus aprendizajes y capacidades de acción, que en la tradicional transmisión de conocimientos atesorados por el profesor. Es decir, requiere abandonar la mirada enciclopedista de la educación para desarrollar una visión atenta a la sociedad de la información, acorde con las exigencias de resolver situaciones problemáticas (Díaz Barriga, 2006).

Así, de acuerdo con Díaz Barriga (2006), el enfoque de competencias puede llegar a mostrar su mayor riqueza si se logra incorporar de manera real en la tarea docente, a través de la promoción de ambientes de aprendizaje.

Ahora bien, sustentar nuestra propuesta en este enfoque no implica ignorar la forma tradicional en que se ha definido la buena docencia. Por ello, se ha incorporado también, como fuente de este marco conceptual, la forma en que se ha medido y definido el concepto en las investigaciones empíricas previas, que tienen una tradición muy anterior y más densa en investigaciones académicas que la formación por competencias.

Finalmente, desde un marco que propone una docencia centrada en el estudiante, resulta imprescindible incluir también la mirada de los propios estudiantes en la definición de qué entenderemos por una docencia de calidad.

De este modo, la conceptualización que se propone desarrollar se basará en tres fuentes diferentes: i) el modelo de Formación por Competencias, ii) los instrumentos actualmente existentes, y iii) la opinión de los estudiantes. 
Asun, R. A., \& Zuñiga, C. (2017). Evaluación docente universitaria: Hacia una perspectiva unificada. Revista de Sociología 32(1), 50-70. doi: $10.5354 / 0719-529 x .2017 .47885$
Con el fin de delimitar la definición de "docencia competente" desde las tres perspectivas elegidas, se utilizaron las siguientes estrategias:

- Las exigencias que el enfoque de formación por competencias hace a los docentes universitarios se han descrito a partir de un análisis de la literatura pertinente (se han revisado 19 producciones: 3 libros, 2 tesis de doctorado y 14 artículos. Para una lista detallada de las publicaciones revisadas, comunicarse con los autores), y de la entrevista individual a tres expertos en el tema, responsables de la implementación de este enfoque en distintas universidades.

- La forma en que usualmente se ha definido y medido la docencia de calidad, se ha identificado a partir de un análisis de contenido cuantitativo de 60 instrumentos, que incluyen 1608 ítems, recopilados a partir de una revisión sistemática de publicaciones en bases de datos especializadas en español e inglés. Las bases revisadas fueron ERIC, ISI, PsycINFO,
PsycARTICLES, EBSCO, ProQuest, Scielo, Redalyc y Dialnet. Los términos de búsqueda empleados fueron: evaluación de la docencia, calidad de la docencia, docencia de excelencia, buen profesor, medición de la docencia, cuestionario de evaluación docente, demandas de los estudiantes, y sus equivalentes en inglés. La revisión incluyó artículos entre 1960 y marzo de 2011 (para una lista detallada de los estudios revisados, comunicarse con los autores).

- Las demandas que los estudiantes hacen a sus docentes, han sido obtenidas a partir del análisis de contenido categorial temático de 15 grupos focales mixtos, realizados a estudiantes de 20 carreras distintas, pertenecientes a seis Facultades, de una universidad pública chilena, durante los años 2008 a 2010. Participaron en estos grupos 89 estudiantes, 41 hombres y 48 mujeres, cuyas edades iban entre los 17 y los 25 años y cursaban entre el primer y el quinto año de sus respectivas carreras.

\section{RESULTADOS}

Demandas del Enfoque de Formación por Competencias

De acuerdo con De Miguel (2005), este modelo plantea que la pedagogía debería centrarse no sólo en transmitir conocimientos (conocer), sino también en entregar destrezas sobre cómo operar (saber hacer) y sobre cómo disponerse personalmente para la ejecución eficiente de las tareas (ser).

$\mathrm{Si}$ bien existen numerosas y disímiles definiciones de competencias, se adoptará como 
Asun, R. A., \& Zuñiga, C. (2017). Evaluación docente universitaria: Hacia una perspectiva unificada. Revista de Sociología 32(1), 50-70. doi: 10.5354/0719-529x.2017.47885

noción general que una competencia es un saber actuar complejo, que se apoya en la movilización y utilización eficaces de una variedad de recursos (internos y externos, actitudinales, valóricos y cognitivos). Se trata de un modelo que se concentra más en el desempeño que en la información (Barriga, 2005) y, por tanto, la evaluación de las actividades pedagógicas debería enfocarse en los logros o resultados del proceso, más que en chequear conocimientos o destrezas fragmentarias (Checchia, 2007).

Ser competente implica entonces combinar una serie de destrezas relacionadas con el ser, el hacer y el conocer. Las más señaladas son las siguientes:

- Del ser: un profesional o docente competente debe conocer y actuar con adecuación a los códigos de conducta, los valores y la ética que regulan su profesión, debe ser responsable con sus obligaciones, tener una correcta imagen de sus capacidades y limitaciones, y debe preocuparse por la calidad de su trabajo.

- Del hacer: en este campo se puede distinguir entre habilidades generales y específicas.

- Habilidades generales: un profesional o docente competente debe ser capaz de integrar distintos conocimientos, planificar y diseñar una tarea, integrar y liderar grupos y proyectos trabajando colaborativamente, tomar decisiones acertadas, pensar creativa y críticamente, actuar en forma autónoma, valorar adecuadamente su trabajo y comunicarse claramente.
Además, debe ser capaz de aprender autónomamente y adaptar su práctica a situaciones diversas.

- Habilidades específicas: un profesional o docente competente debe dominar las herramientas tecnológicas de su disciplina, tener los conocimientos prácticos para ejercer en ese campo y manejar técnicas que le permitan reconocer y resolver los problemas que enfrenta. Esto puede implicar la capacidad de descomponer la situación para afrontarla mejor, y/o ser capaz de mirarla en su conjunto.

- Del conocer: Un docente o profesional competente debe manejar la información teórica disciplinaria necesaria para poder actuar adecuada y eficazmente como tal. Se debe tener un dominio avanzado de dichos contenidos para poder manipularlos $\mathrm{y}$ utilizarlos para fundamentar y reflexionar sobre la práctica realizada. También es relevante comprender, integrar y relacionar los distintos puntos de vista sobre la disciplina, y ser capaz de construir una mirada particular de ella.

A partir de esta teorización, la formación por competencias propone dos cambios en la forma de hacer pedagogía: (a) un cambio en la estructura y forma de administrar el currículum educativo, y (b) un cambio en las conductas del docente de aula. Con relación al primer punto no se profundizará pues no corresponde al objetivo de este artículo. Respecto al segundo, es posible señalar que las 
Asun, R. A., \& Zuñiga, C. (2017). Evaluación docente universitaria: Hacia una perspectiva unificada. Revista de Sociología 32(1), 50-70. doi: 10.5354/0719-529x.2017.47885

nuevas demandas que se hacen al docente universitario son las siguientes:

- El profesor debe tener un claro dominio de los contenidos que va a desarrollar, ser responsable en sus labores docentes, ser capaz de organizar y planificar el curso y tener buena capacidad de transmitir sus saberes. Pero todo esto es secundario en este modelo, ya que se lo considera preexistente en la pedagogía universitaria tradicional. Desde esta perspectiva, la demanda se focaliza más bien en que el profesor actúe como un consultor experto, es decir, el docente debe ser capaz de seleccionar los contenidos, sintetizarlos, ordenarlos y enfatizarlos, para así facilitar que los estudiantes se apropien de ellos, evitando saturarlos con múltiples contenidos presentados como igualmente relevantes.

- El profesor debe tener una buena capacidad de interacción con sus estudiantes y ser hábil en motivarlos al aprendizaje. En este contexto, se hace indispensable que el docente aprenda a desarrollar su capacidad de diálogo y escucha activa, de modo de poder considerar en su docencia los conocimientos, motivaciones y estructuras cognitivas previas de los estudiantes y ser capaz de lidiar con su diversidad. Se espera que el profesor dialogue continuamente con sus alumnos, para conocer la forma en que éstos se representan los contenidos y lograr transmitirles nuevas estructuras de interpretación, más acordes con la mirada de la disciplina.
- Desde el punto de vista de la didáctica, el docente debe manejar diversas metodologías y técnicas de enseñanza, disminuyendo la frecuencia de las clases magistrales, para dar pie a una pluralidad de métodos que pueden facilitar el vínculo entre los conocimientos y la práctica, la comprensión de la pertinencia de lo que se quiere trasmitir, la integración de distintos saberes y el aprendizaje de competencias genéricas.

- En cuanto a la evaluación de los resultados del proceso de enseñanza-aprendizaje, la formación por competencias propone que ésta debe consistir en la demostración de que los alumnos poseen las competencias esperadas. Para ello, las instancias de evaluación deben ser reformuladas, abandonando la hegemonía de las pruebas escritas, para incorporar formatos de múltiple naturaleza (análisis de casos, reportes de investigación, ensayos, presentaciones), que midan aprendizajes teóricos y prácticos, que permitan juzgar la capacidad de integrar conocimientos, que hagan explícitos los procesos a través de los cuales se aprendió y que retroalimenten e involucren a los estudiantes, haciéndolos responsables de sus aprendizajes. Con toda esta información, el docente debe ser capaz de emitir un "juicio experto" global respecto de si el alumno posee o no las competencias que se pretende desarrollar.

- Respecto a los resultados de la formación, los docentes no deben concentrarse exclusivamente en enseñar los conocimientos específicos y las herramientas 
tecnológicas y teóricas de la disciplina, sino que además deben invertir recursos pedagógicos en: i) desarrollar habilidades globales que permitan al estudiante reformular la mirada inicial que tenía de los problemas tratados y construir una nueva y personal estructura cognitiva que le permita investigar, tomar decisiones e intervenir sobre los problemas reales de la profesión; ii) lograr que los estudiantes incorporen los códigos de conducta, los valores, la ética, la responsabilidad, la autoimagen y la preocupación por la calidad, necesarios para ser un buen profesional en el futuro; iii) estimular que el alumno adquiera habilidades genéricas que le permitan integrar distintos conocimientos, reconocer y planificar una tarea, liderar grupos $\mathrm{y}$ proyectos, tomar decisiones, trabajar colaborativamente, pensar creativa y críticamente, actuar en forma autónoma, comunicarse adecuadamente, valorar lo producido, adaptarse al contexto, además de saber aprender. En suma, el docente debe enseñar no sólo contenidos disciplinarios, sino también debe desarrollar habilidades generales del ser y el hacer de la respectiva profesión.

\section{Demandas de la investigación empírica}

Como ya se ha señalado, la tradición de investigación sobre la calidad de la docencia ha construido operacionalmente diversas definiciones de calidad al momento de definir las "áreas" o "dimensiones" que se incluyen en los instrumentos. Pese a que cada uno de los estudios y cuestionarios define de manera distinta estas áreas y a que existen distintas formas de categorizar este conjunto complejo de propiedades, es posible identificar coincidencias entre las diversas propuestas.

Con el fin de develar estas coincidencias, se ha realizado un análisis de contenido de los ítems que conforman los 60 instrumentos encontrados, constatando que todos éstos asumen la forma de escalas tipo Likert.

Los 1608 ítems se agruparon en categorías en función de su contenido, identificándose 48 tipos de ítems que posteriormente se fusionaron en diez grandes temas. Estos diez tópicos constituirían las demandas tácitas que esta tradición investigativa formula a un docente competente:

1. Capacidad de planificación y conducción del curso: agrupa afirmaciones relacionadas con la capacidad del profesor para planificar y desarrollar adecuadamente sus actividades docentes. En cuanto a la planificación, se pregunta por su capacidad para establecer metas y objetivos claros, su habilidad para dar sentido a la asignatura, para exigir una carga de trabajo adecuada, así como para disponer de una buena bibliografía y preparar y actualizar continuamente sus clases. Con relación al desarrollo del curso, se pregunta si el docente cumplió todo el programa y si la metodología del curso fue coherente con los objetivos de aprendizaje señalados en éste.

2. Capacidad de responder a responsabilidades administrativas: se pregunta por el 
Asun, R. A., \& Zuñiga, C. (2017). Evaluación docente universitaria: Hacia una perspectiva unificada. Revista de Sociología 32(1), 50-70. doi: 10.5354/0719-529x.2017.47885

cumplimiento de sus responsabilidades para con la docencia y los estudiantes, como, por ejemplo, i) asistir a todas las clases o, si debe faltar en alguna ocasión, avisar con anticipación y recuperar la clase perdida; ii) respetar los horarios de inicio y término de las sesiones; y iii) retroalimentar las evaluaciones en un tiempo razonable.

3. Dominio de contenidos: se pregunta por el nivel de conocimiento percibido respecto del docente en su área de enseñanza, incluyendo $\mathrm{su}$ desarrollo actual y sus posibles proyecciones. También se consulta sobre la capacidad de sugerir bibliografía complementaria actualizada y de responder adecuadamente las consultas espontáneas de los estudiantes.

4. Capacidad de ser un guía experto: esta dimensión agrupa preguntas relacionadas con la destreza del profesor para ir más allá de una transcripción literal del conocimiento actual sobre la materia, siendo capaz de dar vida a los debates allí contenidos, explicando y haciendo discutir los distintos puntos de vista, relacionándolos con otros temas, incluso fuera del área que se está trabajando, y contextualizándolos con el medio social, cultural e histórico en que esos debates tienen lugar. También se incluyen preguntas relacionadas con la habilidad del profesor para agregar elementos propios a los debates, exponiendo su punto de vista y mostrando su aplicabilidad para la profesión o disciplina. Se destaca también el ser capaz de seleccionar y acentuar las ideas más relevantes, $\quad$ simplificándolas y concentrándose en lo central de los contenidos.

5. Capacidad de comunicación: incorpora los ítems relacionados con la capacidad del profesor para comunicar claramente sus ideas y para ejecutar una serie de conductas verbales y no verbales que potencien que su mensaje llegue a los estudiantes. Dentro de estas habilidades se encuentran la claridad de expresión (que incluye la reiteración de los contenidos más complejos o centrales), la capacidad de ordenación y esquematización de las temáticas, la habilidad de adaptar el ritmo al nivel de los estudiantes, el buen manejo de la voz (volumen adecuado y tonos cambiantes para enfatizar ideas relevantes) y un buen domino del escenario.

6. Capacidad de manejo de diversas metodologías y técnicas de enseñanza: esta dimensión agrupa ítems referidos al uso de recursos didácticos alternativos a la clase expositiva, tales como el trabajo en equipo, el acercamiento a la práctica real por medio de ejercicios y trabajos aplicados y la generación de espacios estructurados de discusión grupal. También se incluye la utilización adecuada de tecnologías de información y comunicación que faciliten la transmisión o aplicación de los conocimientos.

7. Capacidad de motivar a los estudiantes: Contempla la capacidad del profesor para interesar a los estudiantes en los contenidos del curso. Se incluyen características del docente que ayudan a que el mensaje sea bien recibido por los estudiantes, como el 
Asun, R. A., \& Zuñiga, C. (2017). Evaluación docente universitaria: Hacia una perspectiva unificada. Revista de Sociología 32(1), 50-70. doi: 10.5354/0719-529x.2017.47885

entusiasmo, el buen uso del humor, el gusto por la enseñanza, la autoconfianza, el carisma, la capacidad de liderazgo y el asumir un rol activo dentro de la clase. Se incluyen también ítems referidos a si el docente ha sido capaz de plantear a los estudiantes preguntas desafiantes, con el fin de estimularlos intelectualmente y mostrarles la relevancia de los contenidos del curso.

8. Capacidad de generar una interacción eficaz con los estudiantes: en este ámbito, los instrumentos han puesto énfasis en: i) la habilidad de escucha del profesor, que le permita entender la diversidad de sus estudiantes, sus motivaciones y dificultades, para poder adaptar el desarrollo, ritmo y contenidos del curso en función de esa información; ii) la creación de un ambiente de confianza que permita a los estudiantes acudir al profesor en caso de dudas, tanto dentro como fuera de la clase; iii) la capacidad de transmitir preocupación por sus estudiantes, sus aprendizajes y su nivel de comprensión de los temas; y iv) la destreza del profesor para lograr un manejo adecuado del grupo, lo que implica mantener el orden y la disciplina a través del respeto y no del uso de estrategias autoritarias.

9. Capacidad de generar evaluaciones válidas y pertinentes: se considera si los instrumentos utilizados por el profesor miden realmente los aprendizajes logrados, si las evaluaciones son consideradas imparciales y justas, si los instrumentos y la forma de evaluación son congruentes con lo trabajado en clases y si las evaluaciones son transparentes, es decir, si se apoyan en pautas de evaluación conocidas, y existen instancias de discusión de las notas obtenidas por los alumnos. También se incluye acá la capacidad del profesor para utilizar las evaluaciones como instancias de aprendizaje y retroalimentación para el estudiante.

10. Capacidad de desarrollar aprendizajes significativos en conocimientos, habilidades y valores: en esta dimensión se solicita que los alumnos estimen el grado en que han logrado determinados aprendizajes. Es importante notar que cuando se pregunta por los logros de la enseñanza, los investigadores no se limitan a preguntar por los mejoramientos percibidos en conocimiento y comprensión de la temática (aunque esto es lo más frecuente), sino que también se pregunta sobre el fomento del pensamiento propio, de la capacidad de crítica y del desarrollo de capacidades de aprendizaje autónomo y autocrítica. Con menos frecuencia, se encuentran también referencias a la transmisión de valores o consideraciones éticas. 
Asun, R. A., \& Zuñiga, C. (2017). Evaluación docente universitaria: Hacia una perspectiva unificada. Revista de Sociología 32(1), 50-70. doi: 10.5354/0719-529x.2017.47885

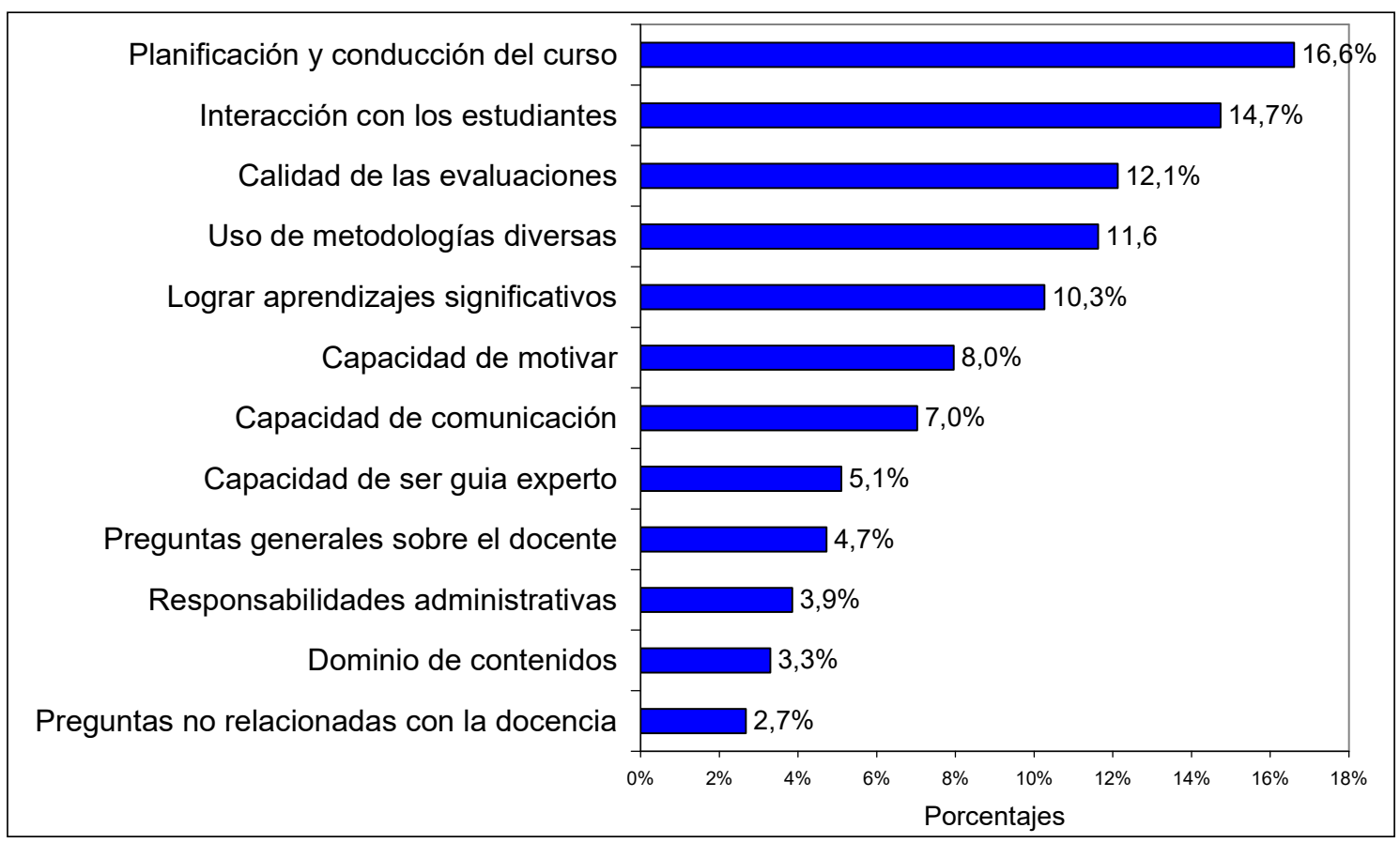

Figura 1. Porcentaje de Ítems según Categorías.

Dado que estas diez categorías o tipos de ítems no aparecen con la misma frecuencia en los instrumentos revisados, se pueden deducir los acentos, énfasis o prioridades a partir del estudio de su frecuencia relativa. Así, en la Figura 1 podemos observar que, en los 1608 ítems codificados, los cinco temas que aparecen con más frecuencia son: planificación y conducción del curso, capacidad de interacción con los estudiantes, calidad de las evaluaciones, uso de metodologías diversas y logro de aprendizajes significativos.

\section{Demandas de los estudiantes}

A partir del análisis de contenido de los grupos focales, es posible señalar que la capacidad de responder a responsabilidades administrativas, la capacidad de planificar y conducir el curso y el dominio de contenidos, constituyen para los estudiantes condiciones mínimas que prácticamente no son dignas de señalar, pues deberían ser cumplidas por todos los profesores (aunque se reconoce que no siempre es así). Por tanto, estas propiedades no alcanzan para definir como "competente" a un profesor.

De manera similar, hay temas que, si bien aparecen en el discurso de los estudiantes, no son especialmente acentuados por ellos, como:

- La didáctica de la enseñanza: los estudiantes demandan, aunque no muy frecuentemente, la utilización de una pluralidad de métodos pedagógicos que sean adecuados a los objetivos de cada clase y la integración de herramientas tecnológicas modernas. No obstante, señalan que los 
profesores que incorporen estas herramientas deben hacerlo adecuadamente, ya que, en ocasiones, por ser mal utilizadas, resultan contraproducentes.

- Aprendizajes significativos: este tema aparece como demanda sólo por parte de alumnos de ciencias sociales y humanidades, quienes esperan que el docente estimule el pensamiento propio, la capacidad crítica y el desarrollo de habilidades de aprendizaje autónomo. El tema del aprendizaje de valores y actitudes, por su parte, es casi inexistente en el discurso de los estudiantes.

En contrapartida, son fuertemente enfatizadas en el discurso estudiantil:

- La capacidad de comunicación, en que se pide (especialmente en los años iniciales) que el profesor no sea monótono, que sea claro para explicar, que reitere las ideas complejas, que sea capaz de ponerse al nivel de sus estudiantes y que sea capaz de esquematizar los contenidos.

- La capacidad de ser un guía experto, en el sentido de que el docente haga discutir distintas posiciones teóricas (evitando mostrar sólo una), agregue su experiencia personal a los contenidos (como investigador o profesional) de manera que las clases no sean simples transcripciones de los textos, que los conocimientos estén contextualizados y actualizados, y que el profesor seleccione y acentúe los elementos centrales.
- La capacidad de generar una interacción positiva con los estudiantes, que implica que el profesor promueva un ambiente de aprendizaje agradable, que les permita hacer consultas, los trate con respeto, facilite su participación dentro de la clase, que esté disponible para resolver sus dudas y que sea capaz de adaptar el curso a su nivel e intereses. Al mismo tiempo, demandan que el docente demuestre preocupación por ellos, sea cercano y mantenga la disciplina sin apelar al autoritarismo.

- La habilidad de motivar a los estudiantes, que implica interesar al estudiante en los contenidos del curso a través de la transmisión del entusiasmo y la pasión que el profesor debe proyectar por las temáticas y por la labor pedagógica.

- La capacidad de generar evaluaciones válidas y pertinentes, con alta relación entre la calificación obtenida y el esfuerzo realizado por el estudiante, y sin sesgos negativos o positivos hacia algunos alumnos. Además, demandan la existencia de mecanismos de retroalimentación de las evaluaciones que les permitan aprender de sus errores (fundamental es que se les brinde un espacio para revisar sus resultados y que exista una pauta de corrección que ellos puedan consultar). También solicitan menos evaluaciones con preguntas cerradas y más preguntas abiertas, para así poder demostrar de mejor manera sus aprendizajes. 


\section{Análisis comparativo}

Recurriendo a las diez categorías que han emergido del análisis de contenido de las escalas analizadas, se ha construido la Tabla 1, que muestra el énfasis que cada fuente otorga a cada uno de esos temas (se han destacado aquellos contenidos acentuados en las respectivas perspectivas).
De manera similar, en la Tabla 2 se presentan los significados que cada una de las perspectivas otorga a esas categorías. Dado que el objetivo es determinar si hay una interpretación diferente ante una misma categoría, sólo se recogen en la tabla los seis temas en que hay al menos dos perspectivas que le prestan alta atención. Se ha destacado la existencia de coincidencias.

Tabla 1. Énfasis diferencial de cada fuente sobre la docencia de calidad

\begin{tabular}{lccc}
\hline & \multicolumn{2}{c}{ Énfasis } \\
\cline { 2 - 4 } & $\begin{array}{c}\text { Formación por } \\
\text { Competencias }\end{array}$ & $\begin{array}{c}\text { Instrumentos } \\
\text { previos }\end{array}$ & Estudiantes \\
\hline Evaluaciones válidas y pertinentes & Alto & Alto & Alto \\
Interacción eficaz con los estudiantes & Alto & Alto & Alto \\
Desarrollo de aprendizajes significativos & Alto & Alto & Medio \\
Manejo de diversas metodologías y técnicas de & Alto & Alto & Medio \\
enseñanza & Alto & Medio & Alto \\
Motivación a los estudiantes & Alto & Bajo & Alto \\
Guía experto & Bajo & Alto & Bajo \\
Planificación y conducción del curso & Bajo & Medio & Alto \\
Capacidad de comunicación & Bajo & Bajo & Bajo \\
Responsabilidades administrativas & Bajo & Bajo & Bajo \\
Dominio de contenidos & & & \\
\hline
\end{tabular}

El análisis de la Tabla 1 permite concluir que, al menos desde el punto de vista de los énfasis temáticos, existe bastante coincidencia entre las tres perspectivas que estudiamos. Así, las dimensiones relacionadas con las evaluaciones y la interacción con los estudiantes son priorizadas por todas las posiciones, mientras que otras cuatro temáticas, son acentuadas por al menos dos de ellas.

No obstante, en la Tabla 2 podemos apreciar que, desde el punto de vista cualitativo, el consenso disminuye. Por ejemplo, tanto la manera en que se entiende la producción de evaluaciones válidas, como el logro de aprendizajes significativos, son bastante disímiles en cada perspectiva. En términos globales, sólo en la dimensión de generar una interacción eficaz con los estudiantes hay un consenso cuantitativo y cualitativo completo entre las tres miradas.

Lo anterior implica que, para construir un enfoque teórico unificado, se debe realizar un proceso que incluya tanto la integración cualitativa de las interpretaciones que hace cada perspectiva, como la asignación de ponderaciones diferentes que permitan 
Asun, R. A., \& Zuñiga, C. (2017). Evaluación docente universitaria: Hacia una perspectiva unificada. Revista de Sociología 32(1), 50-70. doi: 10.5354/0719-529x.2017.47885

priorizar los énfasis de cada una de ellas. siguiente apartado.

Abordaremos este importante desafío en el

Tabla 2. Significado de las dimensiones más utilizadas para conceptualizar la docencia de calidad desde las distintas perspectivas

\begin{tabular}{|c|c|c|c|}
\hline & \multicolumn{3}{|c|}{ Interpretación } \\
\hline & $\begin{array}{l}\text { Formación por } \\
\text { Competencias }\end{array}$ & Instrumentos previos & Estudiantes \\
\hline $\begin{array}{l}\text { Evaluaciones válidas y } \\
\text { pertinentes }\end{array}$ & $\begin{array}{l}\text { Se enfatiza incluir } \\
\text { distintos tipos de } \\
\text { evaluación, } \\
\text { similares al mundo } \\
\text { profesional real }\end{array}$ & $\begin{array}{l}\text { Se enfatiza la } \\
\text { necesidad de } \\
\text { evaluaciones válidas, } \\
\text { transparentes y que } \\
\text { retroalimenten al } \\
\text { estudiante }\end{array}$ & $\begin{array}{l}\text { Se menciona la necesidad de } \\
\text { disminuir el uso de pruebas escritas, } \\
\text { pero en general sólo se demandan } \\
\text { evaluaciones válidas, con } \\
\text { retroalimentación y posibilidad de } \\
\text { apelación. }\end{array}$ \\
\hline
\end{tabular}

Interacción eficaz con los estudiantes

Desarrollo de aprendizajes significativos

Manejo de diversas metodologías y técnicas de enseñanza

Motivación a los estudiantes Guía experto
Se enfatiza la capacidad de escucha, respeto y adaptación a los estudiantes
Se enfatiza la

transmisión de

valores, esquemas

de interpretación y

pautas de

comportamiento

como profesional

autónomo y eficaz
Se enfatiza el

aprendizaje de

contenidos y

débilmente la

transmisión de algunas

pautas de

comportamiento

profesional
Se enfatiza la necesidad de utilizar diversos métodos didácticos, especialmente aquellos que transmitan habilidades profesionales
Existe énfasis parcial en el desarrollo de pensamiento autónomo y crítico
No hay demanda por pluralidad de métodos, sino por el buen uso de las nuevas tecnologías
Se demanda un profesor capaz de interesar a los estudiantes en los contenidos

Se enfatiza la necesidad de que el docente seleccione, ordene, enfatice y haga discutir las distintas temáticas y posiciones 


\section{CONCLUSIONES}

El objetivo de este trabajo es construir una propuesta de conceptualización de la calidad de la docencia universitaria, que facilite el diseño de mejores instrumentos de medición y que considere aportes tanto del modelo de formación por competencias, como de la tradición de investigación empírica y de las demandas de los propios estudiantes.

No obstante, uno de los resultados más relevantes que se desprende de este estudio, y que la literatura no recoge con claridad, es que no existen sólo acuerdos, sino también importantes divergencias entre las diferentes miradas que hemos sistematizado.

Por tanto, la construcción de una perspectiva integrada no es una tarea sencilla. Una de las vías para lograrlo podría ser determinar, desde un punto de vista teórico, la legitimidad que tienen los acentos específicos que cada una de ellas establece. De esta manera se podría decidir si incluir o no una determinada demanda y qué peso asignarle. El problema de esta alternativa es que conduce a una reflexión circular, pues toda afirmación respecto de la legitimidad de una perspectiva sólo puede realizarse a partir de otro enfoque, también situado y parcial, como aquel que se está juzgando.

Por lo tanto, el presente artículo propone una segunda posibilidad: no tomar ninguna decisión respecto de la legitimidad de cada perspectiva y realizar la integración a través de una combinación de los significados que cada una de ellas otorga a las dimensiones en que hemos desagregado la noción de "docente competente". Esta es la propuesta que se presenta en la Tabla 3, donde se integran los sentidos propuestos por las tres perspectivas, en forma de breves descriptores que definen las conductas esperadas de un "docente universitario competente". Así, desde esta nueva perspectiva integrada, se entiende por docente competente aquel que desarrolla una proporción sustantiva de las conductas descritas.

Cabe resaltar que la conceptualización propuesta puede también servir como insumo para el diseño instrumentos de medición de la calidad de la docencia distintos al cuestionario o que vayan dirigidos a otros actores del proceso. De este modo, los descriptores que delimitan a un "docente competente", debidamente seleccionados y adaptados, pueden servir, por ejemplo, como soporte conceptual para estructurar pautas de observación de clases, de análisis de programas de curso, de evaluación por pares, entre otros. 
Tabla 3: Descriptores integrados de un docente competente

$$
\text { Temas }
$$

Planificación y

conducción del

curso

Responsabilidades administrativas

Dominio de contenidos

Guía experto

Capacidad de comunicación

Capacidad de manejo de diversas metodologías y técnicas de enseñanza Motivación a los estudiantes

\section{Contenidos}

- Presenta con claridad los objetivos del curso.

- Programa adecuadamente las actividades y contenidos, con un desarrollo secuencial coherente.

- Propone metodologías adecuadas para cada contenido.

- Propone una carga de trabajo y nivel de dificultad adecuada.

- Cumple con la programación del curso.

- Prepara y actualiza continuamente las clases.

- Prepara bibliografía y material de apoyo adecuado.

- Asiste a clases regularmente y avisa si no puede asistir a alguna sesión.

- Recupera las clases perdidas.

- Respeta los horarios de inicio y término de las clases.

- Corrige las evaluaciones en un tiempo razonable.

- Conoce en profundidad el tema del curso (historia, presente y proyecciones).

- Está actualizado.

- Es capaz de responder preguntas y sugerir bibliografía complementaria.

- Selecciona y acentúa los contenidos clave.

- Expone y discute las distintas perspectivas.

- Aplica los contenidos a situaciones concretas.

- Relaciona los contenidos y teorías, dentro y fuera de la disciplina.

- Expone un punto de vista propio.

- Expone su experiencia como profesional o investigador.

- Se expresa con claridad.

- Realiza esquemas.

- Gradúa la dificultad de los contenidos y reitera los más difíciles.

- Tiene habilidades de expresión (voz, ritmo, gesticulación).

- Utiliza adecuadamente el espacio de la sala.

- Utiliza metodologías diversas.

- Utiliza adecuadamente instrumentos tecnológicos de apoyo.

- Utiliza metodologías adecuadas para cada tipo de aprendizaje.

- Realiza actividades en equipos.

- Utiliza metodologías que permiten aplicar los conocimientos.

- Utiliza metodologías que permiten integrar saberes.

- Genera espacios de participación y discusión grupal.

- Motiva e interesa al estudiante.

- Es capaz de generar una clase entretenida.

- Muestra entusiasmo por los temas.

- Muestra entusiasmo por enseñar.

- Plantea problemas desafiantes.

- Muestra la importancia de los temas tratados. 
Tabla 3: Descriptores integrados de un docente competente (continuación).

\begin{tabular}{|c|c|}
\hline Temas & Contenidos \\
\hline $\begin{array}{l}\text { Interacción eficaz } \\
\text { con los } \\
\text { estudiantes }\end{array}$ & $\begin{array}{l}\text { - Escucha, pregunta y dialoga con los estudiantes. } \\
\text { - Detecta el nivel, diversidad y dificultades de los estudiantes y se ajusta a ellos. } \\
\text { - Se adapta a las demandas de los alumnos. } \\
\text { - Muestra interés por el aprendizaje de los estudiantes. } \\
\text { - Mantiene una relación de respeto con sus educandos } \\
\text { - Mantiene la disciplina sin autoritarismo. } \\
\text { - Crea un ambiente de confianza. } \\
\text { - Brinda apoyo a los estudiantes fuera de la clase. }\end{array}$ \\
\hline $\begin{array}{l}\text { Evaluaciones } \\
\text { válidas y } \\
\text { pertinentes }\end{array}$ & $\begin{array}{l}\text { - Produce evaluaciones que miden adecuadamente los aprendizajes. } \\
\text { - Realiza evaluaciones sin sesgo, trasparentes y verificables (entrega pautas de } \\
\text { corrección). } \\
\text { - Genera evaluaciones congruentes con lo que se ha trabajado. } \\
\text { - Utiliza la evaluación para retroalimentar a los estudiantes. } \\
\text { - Utiliza diversos procedimientos de evaluación. } \\
\text { - Evalúa la reflexión, integración y capacidad de aplicación, más que la } \\
\text { memorización. } \\
\text { - Es capaz de evaluar el proceso de aprendizaje de los alumnos. } \\
\text { - Brinda oportunidades de mejoramiento a los estudiantes. }\end{array}$ \\
\hline $\begin{array}{l}\text { Desarrollo de } \\
\text { aprendizajes } \\
\text { significativos }\end{array}$ & $\begin{array}{l}\text { - Facilita un aprendizaje significativo de los contenidos. } \\
\text { - Facilita la modificación de los esquemas de interpretación inicial de los } \\
\text { estudiantes. } \\
\text { - Facilita el desarrollo del pensamiento crítico y autocrítico. } \\
\text { - Facilita el desarrollo de habilidades de aprendizaje autónomo. } \\
\text { - Facilita el desarrollo de una posición propia ante la temática. } \\
\text { - Logra que los estudiantes apliquen lo aprendido. } \\
\text { - Logra que los estudiantes integren lo aprendido con otros conocimientos. } \\
\text { - Trasmite los principios éticos y valóricos generales y propios de la profesión. } \\
\text { - Enseña como reconocer, planificar, desarrollar y tomar decisiones sobre tareas } \\
\text { - Eomplejas propias de la profesión. } \\
\text { - Enseña como valorar, reflexionar y fundamentar la práctica. } \\
\text { - Enseña como trabajar dentro de grupos y/o liderarlos. } \\
\text { - Enseña las herramientas tecnológicas propias de la disciplina. } \\
\text { - Facilita el desarrollo de una adecuada autovaloración de las capacidades y del } \\
\text { nivel de experticia obtenida. }\end{array}$ \\
\hline
\end{tabular}


Asun, R. A., \& Zuñiga, C. (2017). Evaluación docente universitaria: Hacia una perspectiva unificada. Revista de Sociología 32(1), 50-70. doi: 10.5354/0719-529x.2017.47885

Una pregunta que aún queda por resolver es qué énfasis dar en el instrumento a cada uno de esos temas o dimensiones. Por el momento, y operativamente, lo que se propone es definir la importancia que se quiere otorgar a cada perspectiva y asignar distintas ponderaciones incluyendo un número distinto de afirmaciones para cada tema, en coherencia con la perspectiva seleccionada como prioritaria.

De esta forma, si se quiere producir un cuestionario más cercano al modelo de la formación por competencias, se debería incluir un mayor número de ítems referidos al desarrollo de aprendizajes significativos, la producción de evaluaciones válidas, la buena interacción con los estudiantes, el manejo de diversas metodologías y la capacidad de ser un guía experto. Mientras que, si se desea un cuestionario más cercano a las demandas de los estudiantes, se debe dar mayor peso a la capacidad de comunicación del profesor, por ejemplo.

Queda pendiente una reflexión más profunda que discuta el aporte de cada perspectiva en función de sus méritos para describir de mejor manera a un docente competente, capaz de lograr mejores aprendizajes en sus estudiantes. Finalmente, esperamos que el marco conceptual propuesto en este artículo contribuya a la construcción de instrumentos de evaluación de la calidad de la docencia universitaria teóricamente más sólidos y, por lo tanto, más válidos y confiables.

\section{REFERENCIAS}

Abrami, P., \& D’Apollonia, S. (1991) Multidimensional students' evaluations of teaching effectiveness - generalizability of "N=1" research: comment on Marsh (1991). Journal of Educational Psychology, 83(3), 411-415.

Aleamoni, L. (1999). Student rating myths versus research facts from 1924 to 1998. Journal of Personnel Evaluation in Education, 13(2), 153-166.

Barriga, A. D. (2005). El enfoque de competencias en la educación, ¿una alternativa o disfraz de cambio?. Perfiles Educativos, 28(111), 7 - 36.
Checchia, B. (2007). Estudio y validación de un modelo contextualizado basado en competencias profesionales para la elaboración y valoración de post grados empresariales. Tesis para optar al grado de Doctor, Universidad Complutense de Madrid.

Cohen, P. (1981). Student ratings of instruction and student achievement: A meta-analysis of multisection validity studies. Review of Educational Research, 51, 281-309.

Contreras, G. (2010). Diseño y operación de un sistema de evaluación del desempeño docente con fines formativos: la experiencia de la Pontificia Universidad Católica de 
Asun, R. A., \& Zuñiga, C. (2017). Evaluación docente universitaria: Hacia una perspectiva unificada. Revista de Sociología 32(1), 50-70. doi: 10.5354/0719-529x.2017.47885

Valparaíso, Chile. Revista Iberoamericana de Evaluación Educativa 3, 98-120.

Contreras, G., Faúndez, F., Gutiérrez, A., Jiménez, G. Ponce, M., \& Silva, E. (2007). Diagnóstico del proceso e instrumentos usados actualmente para la evaluación del desempeño docente. En CINDA-MINEDUC. Evaluación del desempeño docente y calidad de la docencia universitaria (pp. 81-98). Santiago de Chile: CINDA - MINEDUC.

D’Apollonia, S., \& Abrami, P. (1997). Navigating student ratings of instruction. American Psychologist, 52(11), 1198-1208.

De Miguel, M. (2005). Modalidades de enseñanza centradas en el desarrollo de competencias: orientaciones para promover el cambio metodológico en el Espacio Europeo de Educación Superior (EEES). Informe de investigación no publicado, Universidad de Oviedo.

Díaz Barriga, Á. (2006). El enfoque de competencias en la educación: ¿Una alternativa o un disfraz de cambio?. Perfiles Educativos, 28(111), 7-36

Elton, L. (1996). Criteria for teaching competence and teaching excelence in higher education. En Aylett, R. y Kenneth, G. (eds.), Evaluating teacher quality in higher education. London - Washington, DC: Falmer Press.

Engelland, B. (2004). Making effective use of student evaluations to improve teaching performance. Journal of Advancement of Marketing Education, 5, 40-46.

García-Garduño, J. M. (2000). ¿Qué factores extraclase afectan la evaluación docente en la educación superior?. Revista Mexicana de Investigación Educativa, 5(10), 303-325.

Gómez, M. A., \& Alzate M.V. (2010). La alegre entrada y el irresistible ascenso de las competencias en la universidad. Educación y Educadores, 13(3), 453 - 474.

Greenwald A., \& Gillmore, G. (1997). Grading leniency is a removable contaminant of student rating. American Psychologist, 52(11), 1209-1217.

Greimel-Fuhrmann, B., \& Geyer, A. (2003). Students' evaluation of teachers and instructional quality - analysis of relevant factors based on empirical evaluation research. Assessment and Evaluation in Higher Education, 28(3), 229 - 238.

Guzman, J. C. (2011). La calidad de la enseñanza en educación superior ¿Qué es una buena enseñanza en este nivel educativo?. Perfiles Educativos, 33 129-141.

Kalayci, N. (2009). The underlying student's reasons for rating set questionnaire items: how student solver the problem of filling out questionnaires. Educational Research Quarterly, 32(4), 36-60.

Marsh, H. (1991). Multidimensional students' evaluations of teaching effectiveness: a test of alternative higher-order structures. Journal of Educational Psychology 83(2), 285-296.

Marsh, H., \& Roche, C. (1997). Making student' evaluations of teaching effectiveness. American Psychologist, 52(11), 1187-1197.

Onwvegbuzie, A., Witcher, A., Collins, K., Filer, J., Wiedmaier, C., \& Moore, G. (2007). Student's perceptions of characteristics of 
Asun, R. A., \& Zuñiga, C. (2017). Evaluación docente universitaria: Hacia una perspectiva unificada. Revista de Sociología 32(1), 50-70. doi: 10.5354/0719-529x.2017.47885

effective college teachers: a validity study of teaching evaluation form using a mixed methods analysis. American Educational Research Journal, 44, 113-160.

Patrick, J., \& Smart, R. (1998). An empirical evaluation of teacher effectiveness: the emergence of three critical factors. Assessment and Evaluation in Higher Education, 32(2), 165-178.

Ruiz, J. (2005). La evaluación de la docencia en los planes de mejora de la Universidad. Educación XXI, 8, 87-102.

Salazar, J. (2008). Diagnóstico preliminar sobre evaluación de la docencia universitaria. Una aproximación a la realidad en las universidades públicas y/o estatales de Chile. Revista Iberoamericana de Evaluación Educativa, 1(3), 67-84.

Salazar, J. (2010). Encuesta de satisfacción estudiantil versus cultura evaluativa de la docencia. Revista Iberoamericana de Evaluación Educativa. 3, 120-132.

Simpson, P., \& Siguaw, J. (2000). Student evaluations of teaching: an exploratory study of the faculty response. Journal of Marketing Education, 22(3), 199-213.
Solar, M. I., \& Díaz, C. (2007). Los procesos de enseñanza-aprendizaje en el aula universitaria: una mirada desde las creencias de académicos de trabajo social y periodismo. Estudios Pedagógicos, 35, 181 197.

Spooren, P., Martelmans, D., \& Denekens, J. (2007). Student evaluation of teaching education: development of an instrument based on 10 Likert-scales. Assessment and Evaluation in Higher Education, 32(6), 667679.

Stake, R., Contreras, G., \& Arbesú, I. (2011). Evaluando la calidad de la universidad, particularmente su docencia. Perfiles Educativos, 33, 155-168.

Tejedor, F. (2003). Un modelo de evaluación del profesorado universitario. Revista de Investigación Educativa, 21(1), 157-182.

Manuscrito recibido: 07-06-2017

Corrección recibida: 18-07-2017

Manuscrito aceptado: 09-08-2017 